\title{
Variation of Ursolic and Betulinic Acid in Five Malus $\times$ domestica clones from Southern Brazil
}

\author{
Andreia C. Wildner $^{1 *}$, Pâmela L. Ferreira ${ }^{1}$, Sendy S. Oliveira ${ }^{2}$, Simone B. Gnoatto ${ }^{1}$, Ana M. Bergold ${ }^{1}$ \\ ${ }^{1}$ Universidade Federal do Rio Grande do Sul/Programa de Pós-graduação em Ciências Farmacêuticas, Av. Ipiranga, 2752, Porto Alegre, RS 90610-000, \\ Brazil. \\ ${ }^{2}$ Universidade Federal do Rio Grande do Sul/Faculdade de Farmácia, Porto Alegre/RS, Brazil.
}

\section{ARTICLE INFO \\ Article history: \\ Received on: 29/10/2017 \\ Accepted on: 12/05/2018 \\ Available online: 30/09/2018}

\section{Key words:}

Triterpene, LC-MS,

ultrasound, apple peel,

ursolic acid, betulinic acid.

\begin{abstract}
An easy, fast and efficient combination of ultrasonic-assisted extraction (UAE) with liquid chromatography-mass spectrometry (LC-MS) was developed for the identification and quantification of ursolic acid (UA) and betulinic acid (BA) in apple peel extract of five clones of Gala and Fuji cultivars ("Baigent", "Fuji Mishima", "Fuji Suprema", "Fuji Select" and "Maxi Gala") from southern Brazil. UA and BA extraction using acetone as a solvent was successfully performed. Chromatographic parameters of the analytical method include positive electrospray ionization (ESI + ) with a flow of $1 \mathrm{~mL}^{-1}$ in isocratic mode consisting of $80 \%$ acetonitrile and $20 \%$ ammonium acetate $10 \mathrm{mM} \mathrm{pH} 6.0$, at room temperature. The method proved to be selective, sensitive, with LOD and LOQ of, 0.087 and $0.266 \mu \mathrm{g} \cdot \mathrm{mL}^{-1}$ for BA, and 0.398 and $2.117 \mu \mathrm{g} \cdot \mathrm{mL}^{-1}$ for UA, linear $(\mathrm{r}>0.99)$, precise, accurate and robust, for all analytes. The optimization of UAE combined with the LC-MS technique allowed completing the procedure (extraction and analysis) in less than $4 \mathrm{~h}$. These methods were applied for the first-time characterization of five clones. The results demonstrated that the UAE-LC-MS method is applicable to the routine analysis of triterpene acids in apple peel.
\end{abstract}

\section{INTRODUCTION}

Ursolic acid (3ß-Hydroxy-12-ursen-28-ic acid, UA) and betulinic acid (3ß-Hydroxy-20 (29)-lupaene-28-oic acid, $\mathrm{BA})$ are natural pentacyclic triterpenes (PCT), whose importance and a wide diversity of pharmacological properties have remained extremely attractive for scientific research. Recently, these compounds were highlighted to serve as scaffolds for the semisynthesis of new lead bioactive agents, due to a remarkable chemistry structure (Broniatowski et al., 2014; Hsu et al., 2015; Kalani et al., 2013; Kashyap et al., 2016). From the biological perspective, these compounds are mainly linked to antimalarial, anticancer, anti-inflammatory and antidiabetic activities (Bero et al., 2013; Jang et al., 2014; Li et al., 2015; Mitsuda et al., 2014; Silva 2015b).

"Corresponding Author

Andreia C. Wildner, Universidade Federal do Rio Grande do Sull

Programa de Pós-graduação em Ciências Farmacêuticas, Av. Ipiranga, 2752, Porto Alegre, RS 90610-000, Brazil.

E-mail: andreiacwcl@gmail.com
Although UA and BA are secondary metabolites that occur widely on surfaces such as cuticle waxes and stem barks of many plants, apple peel has been frequently recommended as an important source to obtain triterpene compounds (up to $50 \%$ of dry weight) (Mazumder et al., 2013; Thilakarathna et al., 2013). For apples, besides differences in appearance, texture, and flavor, there are significant variations between different cultivars in the concentration and composition of these bioactive compounds (McGhie et al., 2012). In Brazil, apples (Malus $\times$ domestica) are among the main species of national fruticulture, where the Gala and Fuji cultivars predominate. Genetic alterations, spontaneous and selected for some agronomic advantage, such as the color of apple peel gave rise to "clones". Currently, the main mutant clones of these varieties are: 'Baigent' ('Brookfield'), 'Maxi Gala', 'Fuji Supreme', 'Fuji Select' and 'Fuji Mishima', whose production is concentrated in the south of the country (Paraná, Santa Catarina, and Rio Grande do Sul) (EMBRAPA, 2013) (Figure 1).

Considering the importance of these bioactive compounds for pharmaceutical purposes, this work presents, the development of a practical and reliable analytical method for the 
extraction, identification, and quantification of UA and BA in apple peel extracts (APE) using LC-MS. This method was validated to ensure efficient application in the quality control of extracts. An additional goal of this study was to evaluate the composition of each clone, in terms of UA and BA content, considering, the importance of having abundant and sustainable natural sources available for future obtention of these bioactive compounds.

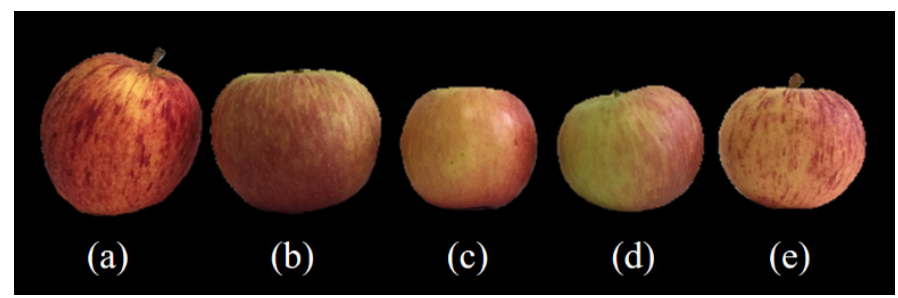

Fig. 1: Apple clones from Gala and Fuji cultivars, (a) Baigent, (b) Fuji Mishima, (c) Fuji Suprema, (d) Fuji Select and (e) Maxi Gala.

\section{MATERIALS AND METHODS}

\section{Chemicals and reagents}

Acetonitrile and acetic acid were HPLC grade, purchased from Sigma-Aldrich (St. Louis, MO, USA). Reagent grade ammonium acetate and acetone were obtained from Merck (Darmstadt, Germany). Ultra-pure water (Milli-Q, Millipore, USA) was used to prepare all the solutions.

Analytical standards of ursolic acid, betulinic acid, and spironolactone (internal standard) were purchased from SigmaAldrich (St. Louis, MO, USA). The purity of all standards was at least $95 \%$.

\section{Preparation of reference solutions}

The stock solution was prepared to contain $100 \mu \mathrm{g} \cdot \mathrm{mL}^{-1}$ of each analyte, diluted in methanol $80 \%$ (v/v in water), named diluent solution (DS). Working standard solutions were prepared to contain $10 \mu \mathrm{g} \cdot \mathrm{mL}^{-1}$ of each analyte, by diluting stock solution. All solutions were kept protected from Light and stored at $4{ }^{\circ} \mathrm{C}$.

\section{Fruit material}

Apples of the Gala and Fuji cultivars were kindly provided by EMBRAPA Uva e Vinho, Bento Gonçalves/RS (Brazil) and harvested in March 2017. A total of five clones: Gala Baigent, Fuji Mishima, Fuji Select, Fuji Suprema and Maxi Gala were harvested in Vacaria (Brazil) and used in the study (Fig. 1).

\section{Gravimetric analysis}

Before the study began, the water content in the apple peel samples was determined according to the USP 40, Gravimetric Method for Articles of Botanical Origin. About $3 \mathrm{~g}$ of apple peel from each Gala and Fuji cultivar were accurately weighed in a tared evaporating flask. The samples were dried in an oven set at $105^{\circ} \mathrm{C}$ for $5 \mathrm{~h}$ and weighed. Then, at $1 \mathrm{~h}$ intervals, the flasks were quenched in desiccators at room temperature and weighed again. After another $3 \mathrm{~h}$ in the oven, the difference between two successive weightings corresponds to no more than $0.25 \%$ and the test was ended. The gravimetric analysis was performed in triplicate for each cultivar.

\section{Preparation of apple peel extracts (ape)}

Each fruit was washed and then cut into slices $(\sim 0.5$ $\mathrm{cm}$ ) and peeled. The fresh peels were taken to a knife mill to reduce them to small pieces of apple peel. Approximately 3.0 $\mathrm{g}$ of each clones' peel were homogenized with $15 \mathrm{~mL}$ acetone and the mixture was left to extract BA and UA, in an ultrasonic bath (Model Sonic Clean 15, Sanders Medical, Brazil), at room temperature, for 60 minutes. The supernatant was collected and the volume was completed to the initial level. The mixing was done in a vortex for $10 \mathrm{~s}$ and then filtered through a $0.22 \mu \mathrm{m}$ Nylon membrane (Allcrom, Brazil) and diluted in methanol (1:1) before injection. Three cycles of this procedure were performed in order to obtain three extractions for each sample. All the yields and compositions were calculated on a moisture-free basis and determined as described in section ("Gravimetric Analysis") and represent the mean values of three experiments.

\section{Apparatus}

Agilent 1260 infinity LC system equipped with a G1311B quaternary pump, a G1329B autosampler, G1314F UV/ VIS detector, G1316A thermostatizer coupled to an Agilent 6120B series mass detector and a Chemstation (v. B.04.03) software were used (Palo Alto, CA, USA). Column C18 RP (150 mm $\times 4.6 \mathrm{~mm}$ i.d., $5.0 \mu \mathrm{m}$ particle size) (Torrance, CA, USA) was maintained at room temperature. Flow rate at $1.0 \mathrm{~mL} \cdot \mathrm{min}^{-1}$ in an isocratic condition consisting of $80 \%$ solvent A (acetonitrile), $20 \%$ solvent $\mathrm{B}$ $10 \mathrm{mM}$ (ammonium acetate $\mathrm{pH}$ 6.0) was used. The injected volume was $10 \mu \mathrm{L}$. Single-stage mass spectrometer detector was operated with electrospray ionization source in positive mode (ESI + ) and the following parameters were set for the spray chamber: capillary voltage $4000 \mathrm{~V}$, drying gas flow $12 \mathrm{~L} \cdot \mathrm{min}^{-1}$, nebulizer pressure 50 psig and drying gas temperature $350^{\circ} \mathrm{C}$. The gain value was kept at 1 . Parameters were set to optimize the quantification ion, and analyses were performed in single ion monitoring (SIM) mode for each analyte $(\mathrm{AU}, \mathrm{AB})$ with fragmentor values set in 120 for triterpene acid and 100 for IS.

\section{Method validation}

The analytical method was validated, according to the performance characteristics defined in the guidelines $(\mathrm{ICH}$, 2005).

Selectivity was assessed comparing retention times (Rt) and the molecular mass of BA and UA standard with a pool of APE and also by spiking the sample with $1.0 \mu \mathrm{g} \cdot \mathrm{mL}^{-1}$ of standard.

Calibration curves were constructed for each sample with 6 concentration levels on 3 different days, evaluating the correlation coefficients ( $r$ ) and standardized residual plots to check outliers that were eliminated if found to be outside the limit ( \pm 3 standard deviations). The chromatographic profile of APE is characterized by a high difference of peak area between the analytes. Concentrations of BA ranged 0.5, 1.0, 1.5, 2.0, 3.0 and $4.0 \mu \mathrm{g} . \mathrm{mL}^{-1}$ and UA range was 5.0, 10.0, 25.0, 50.0, 75.0 and $100.0 \mu \mathrm{g} \cdot \mathrm{mL}^{-1}$. Linearity was evaluated by ANOVA and linear regression analyses.

Sensitivity was assessed by determination of LOD (limit of detection) and LOQ (limit of quantification). They were estimated theoretically. The factors 3.3 and 10 for the detection and quantitation limits, respectively, were multiplied by the 
ratio from the standard deviation of the intercept and, the slope according to the guidelines (ICH, 2005).

Precision was assessed by an intra-day test (repeatability) and an inter-day test (intermediate precision). Relative standard deviation (RSD) of BA and UA content (\%) in the extract was used to express precision. To perform the repeatability study, six replicate experiments on an average concentration $\left(1.5 \mu \mathrm{g} \cdot \mathrm{mL}^{-1}\right.$ for BA) and ( $50.0 \mu \mathrm{g} \cdot \mathrm{mL}^{-1}$ for UA) were carried out on the same day. Intermediate precision was measured by comparing the results of the assay on different days.

Accuracy was determined by recovery study and carried out by spiking the extract with 3 concentration levels (low, medium, and high), for each analyte. Concentrations used were 1.0, 1.5, and $2.0 \mu \mathrm{g} . \mathrm{mL}^{-1}$ for BA; and 25.0, 35.0, and 40.0 $\mu \mathrm{g} . \mathrm{mL}^{-1}$ for UA. These analyses were conducted in triplicate and processed on the same day. The results were estimated through comparison between theoretical concentrations (application of an analytical procedure to reference standard of known purity) and the experimentally measured concentrations.

Robustness was evaluated by alterations in three factors of the analytical method: a) the proportion of organic solvent in the mobile phase, using acetonitrile and ammonium acetate aqueous, $10 \mathrm{mM}$, at $(90: 10 \mathrm{v} / \mathrm{v}, 80: 20$ and $70: 30 \mathrm{v} / \mathrm{v}) ; b)$ flow rate $\left(0.8,1.0\right.$ and $\left.\left.1.1 \mathrm{~mL} . \mathrm{min}^{-1}\right) ; c\right)$ capillary voltage employing $(3000$, 4000 and $5000 \mathrm{~V})$. Each factor was evaluated separately and the content (\%), Rt and specificity were used to estimate the influence on the method. Results are express in terms of RSD\%, over UA and BA content $(\%)$.

Extract stability was assessed by monitoring each analyte content (\%) along the experimental sections. A fresh sample of the extracts was prepared daily.

Statistical analyses were performed through ANOVA and Linear Regression using Excel 2013 software (Microsoft Corp., Redmond, United States); Tukey's test was performed using Minitab® 18.1 (Minitab, Inc., in the United States).

\section{Method application}

A validated method was applied to the analyses of APE obtained from 5 clones (Figure 1), for the purpose of evaluating their efficiency and reliability. Three apples from each clone were used to prepare a pool from each clone were used to prepare a pool. The APE was prepared as described in section ("Preparation of Apple Peel Extracts (APE)'). All analyses were conducted in triplicate and analyte averages from the extract were compared with the corresponding standards in terms of $\mathrm{Rt}$ and $\mathrm{m} / \mathrm{z}$ of the molecular ion. For all analyses, the dry mass of apple peel was used as the basis of the assay experiment, considering the average water content, according to the Gravimetric Analysis.

\section{RESULTS AND DISCUSSION}

The purpose of the study is to develop an efficient, simple and fast methodology focused on the detection and quantification of UA and BA in APE for application in a quality control routine. Thus, the first goal of this study is to report a methodology that combines techniques of extraction and analysis by LC-MS, in less than 4 hours. The second goal of this study is to produce the first profile of UA and BA on five clone's Gala and Fuji cultivar ("Baigent", "Fuji Select", "Fuji Mishima", "Fuji Suprema" and
"Maxi Gala") from the South of Brazil.

\section{LC-MS method optimization}

Chromatographic conditions of analytical method conditions were chosen by testing different solvents (acetonitrile and methanol), $\mathrm{pH}$ modifiers (acetic acid, ammonium acetate) and ionization mode. The results allowed the following considerations: i) mixture of methanol and water in different proportions was not effective for the detection of BA and UA in the matrix; ii) higher percentage of acetonitrile promoted the detection with high-intensity peaks, good resolution among the UA and BA and faster run; iii) acetic acid as the mobile phase modifier at $\mathrm{pH} 3.0$ caused loss of reproducibility in the peak area $(\mathrm{CV} \%, 20) . i v)$ the addition of buffer at low concentration $(0.01 \mathrm{M}$ ammonium acetate) ensured the complete ionization of molecules during the analyses and eliminated oscillation in the peak areas; $v$ ) at $\mathrm{pH} 3.0$, the ionization intensity of the analyte is poor, based on UA and BA $\mathrm{pKa}(\sim 4.0)$, thus raising to $\mathrm{pH} 6.0$, the sensitivity was improved significantly; $v i)$ in negative mode ionization the triterpene acids could not be detected; vii) the detection was achieved in positive polarity, where the isomers (BA and UA) exhibited the same three MS signals ( $m / z$ 457, 439 and 479), which differed in terms of relative intensity, for each analyte. The signal $\mathrm{m} / \mathrm{z} 457$ is recognized as the molecular ion, and confirmation ion $\mathrm{m} / z 439$ is associated with loss of water and $\mathrm{m} / \mathrm{z} 479$ correspond to molecular ion plus a sodium adduct (Kamel et al., 1999; Rhourri-Frih et al., 2009). So, in order to avoid the influence of solvent variation, which could be impacted with MS ion source during the triterpene analysis, the isocratic elution mode was maintained (De Oliveira et al., 2002). (Supplementary Material Figures S1-S2. Finally, the high sensibility of MS, allowed the more accurate detection of all analytes in APE, especially for BA, which appeared in a smaller amount in APE. The optimal chromatographic condition was established and a typical chromatogram obtained by applying the analytical method described is shown in Figure 2.

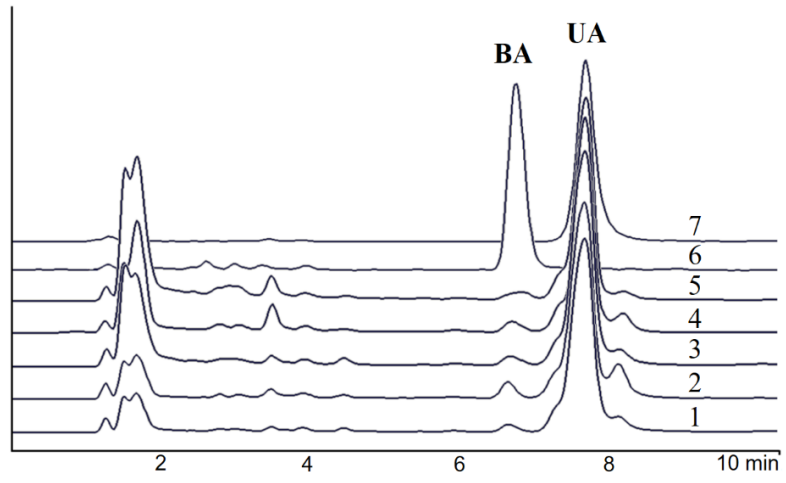

Fig. 2: Representative LC-MS chromatogram (SIM mode) of the APE and standard solution of UA and BA at $m / z$ 457. Clones "Fuji Suprema" (1), "Fuji Mishima" (2), "Fuji Select" (3), "Baigent" (4), "Maxi Gala" (5), BA standard solution (6) and UA standard solution (7).

\section{LC-MS Method validation}

Once the efficient chromatographic separation was achieved, with no co-eluted peaks, the selectivity could be determined. The comparison with the standard solution was used for identification, (Figure 2). The compounds of interest in APE, 
showed an Rt corresponding to the authentic standard solution, with (6.70 min, BA) and (7.59 min, UA). All compounds were displayed in MS-spectra with an $[\mathrm{M}+\mathrm{H}]^{+}$ion at $\mathrm{m} / z 457.0$ corresponding to the molecular ion formula $\mathrm{C}_{30} \mathrm{H}_{48} \mathrm{O}_{3}$. (Supplementary Material Figures S2-S3).

Table 1: Calibration results of the LC-MS method for pentacyclic triterpene acid.

\begin{tabular}{cccc}
\hline Calibration* & Recomendation & Betulinic Acid & Ursolic Acid \\
\hline Range $\left(\mu \mathrm{g} \cdot \mathrm{mL}^{-1}\right)$ & - & $0.5-4.0$ & $5.0-100.0$ \\
Rt $(\mathrm{min}) \pm \mathrm{SD}$ & - & $6.41 \pm 0.05$ & $7.25 \pm 0.06$ \\
$r$ & $>0.99$ & 0.9990 & 0.9996 \\
Slope $\pm \mathrm{SD}$ & - & $70872 \pm 261.8$ & $20962 \pm 766.5$ \\
Intercept $\pm \mathrm{SD}$ & - & $35891 \pm 3269.9$ & $267287 \pm 21857.9$ \\
Linear regression & $>0.93$ & 919.2 & 662.4 \\
Linearity deviation & $<.4$ & 3.11 & 2.03 \\
LOD $\left(\mu \mathrm{g} \cdot \mathrm{mL}^{-1}\right)$ & - & 0.087 & 0.398 \\
LOQ $\left(\mu \mathrm{g} . \mathrm{mL}^{-1}\right)$ & - & 0.266 & 2.117 \\
\hline
\end{tabular}

*Duplicate of calibration curves, on three different days. Calculations were performed according to ICH (2005).

${ }^{* *}$ Calculated by least squares regression, with $\mathrm{p}<0.01(\mathrm{n}=18)$.

Linearity was observed for UA and BA, over a sixpoint linear calibration curve covering the expected range for each analyte in the APE, with an adequate coefficient ( $\mathrm{r}>0.99)$. ANOVA confirmed a linear relationship (Fcalc $>$ Ftab, $p<$ 0.01 ) and no deviation from Linearity (Fcalc $<$ Ftab, $p<0.01$ ). The residual coefficient was also evaluated and the results were acceptable. Theoretical LOQ and LOD were calculated based on system performance and equations $(\mathrm{y}=20962 \mathrm{x}+267287$, UA) and $(y=70872 x+35891$, BA $)$, although lower LODs and LOQs were technically possible by the experimental assay. Linearity, LOD, and LOQ for each analyte are presented in the calibration curve data (Table 1). Linearity curve of the two analytes (UA and BA) are shown in Supplementary Figure S1.

Precision, accuracy, and robustness are summarized in Table 2. Dilution integrity was evaluated, assuring the possibility of performing analyses down to a concentration of $0.1 \mu \mathrm{g} \cdot \mathrm{mL}^{-1}$ with acceptable precision and accuracy. Repeatability and interday precisions were acceptable because the \%RSD between determinations did not exceed $2.07 \%$. Some deviation could only be observed only for BA, probably affected by the matrix, due to its low concentration compared with UA. Accuracy was demonstrated by suitable recoveries under a range (from 95 to $105 \%$ ), as prescribed by the ICH (2005). The developed method was accurate and demonstrated no remarkable matrix effect. Evaluation of robustness), the small modifications in the original system, did not produce a significant effect on the analyte content, (RSD\% less than 5\%). Changing the flow rate, some Rt values were modified. The replacement of the chromatographic column (Agilent ${ }^{\circledR}$ Poroschell 120 EC-C18, $4.6 \times 50$ mm, $2.7 \mu \mathrm{m}$ ) equipped with guard column $\mathrm{C} 18 \mathrm{RP}$, did not involve changing the retention times and the contents remained in the original condition. Thus, the method was considered robust enough to be included in the Quality Control routine.

\section{Extraction method}

Liquid-liquid extraction (LLE) assisted by UAE at room temperature was employed, due to the low consumption of organic solvents and the short time required, when compared with microwave and Soxhlet extraction (data not shown) (Fan et al., 2016). LLE conditions using different solvents with polar or intermediate polarity (acetonitrile, methanol or acetone) and a less polar one (ethyl acetate or dichloromethane) were tested and allowed some observations: $i$ ) non-polar solvents do not extract the pentacyclic triterpenes (Bars-Cortina et al., 2017); ii) the use of acetonitrile reduces the extraction percentage, decrease peak area and poor detection of BA; iii) the use of $100 \%$ methanol reduces peak symmetry; $i v$ ) the use of only acetone allowed the detection of UA and BA, with very good intensity and selectivity, however, due to the high volatility of acetone, before the LC-MS analyses, the mixture was diluted in methanol (1:1). The optimal condition for the extraction method includes UAE in acetone with 3 cycles of 60 minutes, followed by dilution in methanol (1:1) (Wójciak-Kosior et al., 2013). These results combined with Tukey's test analyses demonstrate that for the proposed extraction method 3 cycles are necessary for the efficient removal of the acid triterpenes from the matrix. Extraction for a longer time resulted in insipid contents of UA and BA. The average content of the analytes obtained through 3 extraction cycles performed in triplicate is shown in Figure 3.

Table 2: Precision, accuracy, and robustness of the LC-MS method for pentacyclic triterpene acids in apple peel extract.

\begin{tabular}{ccc}
\hline Parameters & Betulinic Acid & Ursolic Acid \\
\hline Repeatability (\%RSD) & 1.61 & 1.16 \\
Intermediate precision (\%RSD) & 2.07 & 1.63 \\
\hline Accuracy (\%recovery) & & \\
\hline Low & $95.25-100.50$ & $95.93-99.88$ \\
Media & $96.16-105.33$ & $99.97-102.37$ \\
High & $97.51-100.69$ & $96.60-98.91$ \\
\hline Robustness & & 3.50 \\
\hline Flow (\%RSD) & 3.82 & 2.31 \\
Capillary Voltage (\%RSD) & 4.24 & 3.83 \\
Mobile Phase (\%RSD) & 3.92 &
\end{tabular}

*\% RSD: in terms of the content of each analyte of interest.

\section{LC-MS analysis of apple peel extracts}

Once optimized and validated, the LC-MS method developed was applied to the quantification of UA and BA in APE from five clones of Gala and Fuji cultivars aiming to investigate which of the clones would be richer in pentacyclic triterpenes. APE profiling by LC-MS revealed the presence of UA and BA in all studied clones (Figure 2).

Clones from Gala and Fuji cultivars contain a significant amount of PCT and the target varieties can be characterized by the high concentration of UA in comparison to the BA concentration. UA stands out as the main compound with concentrations ranging from $(0.073-1.897 .8 \% \mathrm{DW})$. BA was also found but to a lesser extent $(0.004-0.166 \%$ DW). These concentrations are in agreement with literature reported for Malus species. (Andre et al., 2012; Bars-Cortina et al., 2017; Farneti et al., 2015; Jäger et al., 2009; Zhang et al., 2014). In terms of the total content of UA and BA, the ranking is: 'Fuji Select' $>$ 'Fuji Suprema' $>$ 'Fuji Mishima' > 'Baigent' > 'Maxi Gala' (Figure 3). 
Significant differences were observed in the total average of UA content among the studied clones. In special for BA, that shows drastic variation among the clones and the 'Maxi Gala' exhibits the highest amount of it. In addition, a large content of the compounds of interest was found in clones from the Fuji cultivar.
According to Andre et al. (2012), fruits which are characterized by a thicker opaque and waxy skin tend to have higher triterpene acid contents. Considering the above, these findings contribute to outlining the first profile of the triterpene acids (UA and BA) content of Gala and Fuji clones originating in South Brazil.
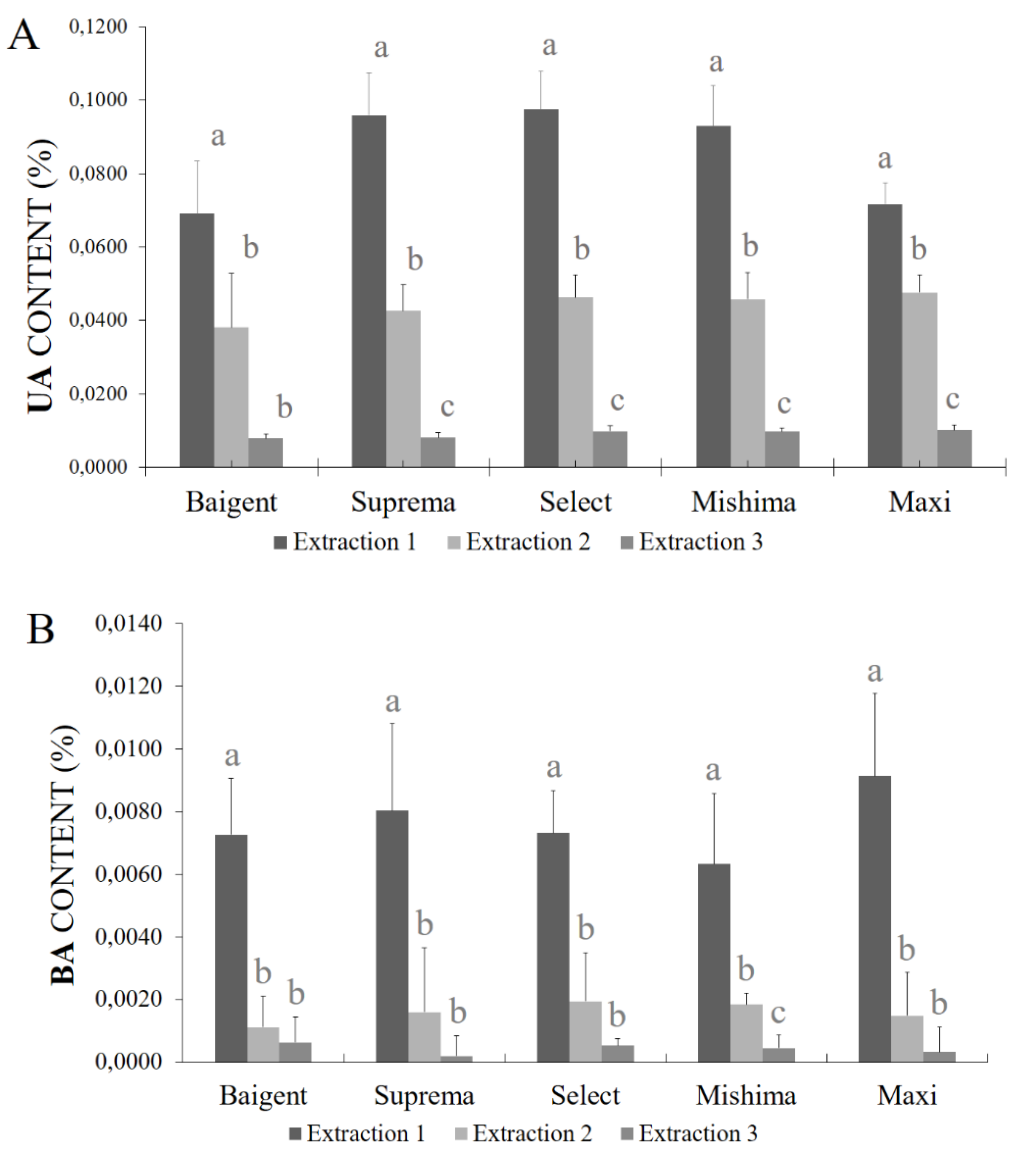

Fig. 3: Pentacyclic triterpene acid content in apple peel of five clones from Gala and Fuji cultivars according to the analytical method developed. (A) Ursolic acid; (B) Betulinic acid, calculated based on a moisture-free basis and representing the average values of three experiments and expressed as $\mu \mathrm{g}$. $\mathrm{g}^{-1}, \mathrm{DW}$. Different letters indicate significant differences between clones PCT content according to Tukey's test $(P<0.05)$.

\section{CONCLUSION}

The present work developed an efficient, sensitive and rapid method that combines techniques of extraction, detection, and quantification by UAE-LC-MS for analysis of UA and BA in apple peel extracts of $M . \times$ domestica, with the advantage of requiring less than $4 \mathrm{~h}$ for the total process and yields compatible with those reported in the literature. LC-MS provided the confirmation of analytes in five clones from Gala and Fuji cultivars ("Baigent", "Fuji Select", "Fuji Mishima", "Fuji Suprema" and "Maxi Gala") from South Brazil, besides contributing to sketching the first profile of these bioactive compounds in the mentioned samples. The goal of the study to develop and validate a reliable tool for the determination of UA and BA in apple peel extracts, aiming to identify the best source of these bioactive compounds was successfully achieved and also proved suitable for routine quality control of similar matrices.

\section{ACKNOWLEDGMENTS}

The authors want to thank CAPES (Coordenação de Aperfeiçoamento de Pessoal de Nível Superior), PPGCFUFRGS (the Pharmaceutical Sciences Graduate Program-Federal University of Rio Grande do Sul) for the financial support, Dr. Paulo Ricardo Dias de Oliveira from Embrapa Uva e Vinho for the time, clarifications and the donation of apple samples. Authors also thank the team of LAPS (Laboratório de Padrões Secundários) for their collaboration.

\section{CONFLICT OF INTERESTS}

There are no conflicts of interest.

\section{REFERENCES}

Andre CM, Greenwood JM, Walker EG, Rassam M, Sullivan M, Evers D, Perry NB, Laing WA. Anti-inflammatory procyanidins and triterpenes in 109 apple varieties. J Agric Food Chem, 2012; 60:1054610554.

Bars-Cortina D, Macià A, Iglesias I, Romero MP, Motilva MJ. Phytochemical Profiles of New Red-Fleshed Apple Varieties Compared with Traditional and New White-Fleshed Varieties. J Agric Food Chem, 2017; 65:1684-1696. 
Bero J, Hérent MF, Schmeda-Hirschmann G, Frédérich M, Quetin-Leclercq J. In vivo antimalarial activity of Keetia leucantha twigs extracts and in vitro antiplasmodial effect of their constituents. J Ethnopharmacol, 2013; 149:176-183.

Broniatowski M, Flasiński M, Ziẹba K, Miśkowiec P. Interactions of pentacyclic triterpene acids with cardiolipins and related phosphatidylglycerols in model systems. Biochim Biophys ActaBiomembr, 2014; 1838:2530-2538.

De Oliveira BH, Santos CAM, Espíndola APDM. Determination of the triterpenoid, betulinic acid, in Doliocarpus schottianus by HPLC Phytochem Anal, 2002; 13:95-98.

Fan J-P, Liao D-D, Zhang X-H. Ultrasonic assisted extraction of ursolic acid from apple pomace: A novel and facile technique. Sep Sci Technol, 2016; 51:1344-1350.

Farneti B, Masuero D, Costa F, Magnago P, Malnoy M, Costa $\mathrm{G}$, Vrhovsek U, Mattivi F. Is there room for improving the nutraceutical composition of apple? J Agric Food Chem, 2015; 63:2750-2759.

Hsu T-I, Chen Y-J, Hung C-Y, Wang Y-C, Lin S-J, Su W-C, Lai M-D, Kim S-Y, Wang Q, Qian K, Goto M, Zhao Y, Kashiwada Y, Lee K-H, Chang W-C, Hung J-J. A novel derivative of betulinic acid, SYK023, suppresses lung cancer growth and malignancy. Oncotarget, 2015; 6:1367113687.

ICH. 2005. Harmonised Tripartite Guideline; Validation of Analytical Procedures: Text and Methodology. Geneva: IFPMA.

Jäger S, Trojan H, Kopp T, Laszczyk MN, Scheffler A. Pentacyclic triterpene distribution in various plants - rich sources for a new group of multi-potent plant extracts. Molecules, 2009; 14:2016-2031.

Jang SE, Jeong JJ, Hyam SR, Han MJ, Kim DH. Ursolic acid isolated from the seed of cornus officinalis ameliorates colitis in mice by inhibiting the binding of lipopolysaccharide to toll-like receptor 4 on macrophages. J Agric Food Chem, 2014; 62:9711-9721.

Kalani K, Agarwal J, Alam S, Khan F, Pal A, Srivastava SK. In Silico and In Vivo Anti-Malarial Studies of $18 \beta$ Glycyrrhetinic Acid from Glycyrrhiza glabra. PLoS One, 2013; 8:1-11.

Kamel AM, Brown PR, Munson B. Effects of mobile-phase additives, solution $\mathrm{pH}$, ionization constant, and analyte concentration on the sensitivities and electrospray ionization mass spectra of nucleoside antiviral agents. Anal Chem, 1999; 71:5481-5492.

Kashyap D, Tuli HS, Sharma AK. Ursolic acid (UA): A metabolite with promising therapeutic potential. Life Sci, 2016; 146:201213.

Li D, Du Z, Li C, Liu Y, Goodin S, Huang H, He Y, Zhang Y, Wang $\mathrm{H}$, Zheng $\mathrm{X}$, Zhang $\mathrm{K}$. Potent inhibitory effect of terpenoids from
Acanthopanax trifoliatus on growth of $\mathrm{PC}-3$ prostate cancer cells in vitro and in vivo is associated with suppression of NF- $\mathrm{KB}$ and STAT3 signalling. J Funct Foods, 2015; 15:274-283.

Mazumder K, Tanaka K, Fukase K. Cytotoxic activity of ursolic acid derivatives obtained by isolation and oxidative derivatization. Molecules, 2013; 18:8929-8944.

McGhie TK, Hudaul, S, Lunken RCM, Christeller JT. Apple peels, from seven cultivars, have lipase-inhibitory activity and contain numerous ursenoic acids as identified by LC-ESI-QTOF-HRMS. J Agric Food Chem, 2012; 60:482-491.

Mitsuda S, Yokomichi T, Yokoigawa J, Kataoka T. Ursolic acid, a natural pentacyclic triterpenoid, inhibits intracellular trafficking of proteins and induces accumulation of intercellular adhesion molecule-1 linked to high-mannose-type glycans in the endoplasmic reticulum. FEBS Open Bio, 2014; 4:229-239.

Rhourri-Frih B, Chaimbault P, Claude B, Lamy C, André P, Lafosse M. Analysis of pentacyclic triterpenes by LC-MS. A comparative study between APCI and APPI. J Mass Spectrom, 2009; 44:71-80.

Silva FRMB. The mechanism of action of ursolic acid as insulin secretagogue and insulinomimetic is mediated by cross-talk between calcium and kinases to regulate glucose balance. Biochim Biophys Acta, $2015 ; 1850: 51-61$.

United State Pharmacopeia. USP 40. Physical tests / <921> Water determination. Method III. Procedure for Articles of Botanical Origin. [ONLINE] Available at: https://hmc.usp.org/sites/default/files/ documents/HMC/GCs-Pdfs/c921.pdf [Accessed 26 April 2018].

Thilakarathna SH, Rupasinghe HPV, Needs PW. Apple peel bioactive rich extracts effectively inhibit in vitro human LDL cholesterol oxidation. Food Chem, 2013; 138:463-470.

Wójciak-Kosior M, Sowa I, Kocjan R, Nowak R. Effect of different extraction techniques on quantification of oleanolic and ursolic acid in Lamii albi flos. Ind Crops Prod, 2013; 44:373-377.

Zhang S, Sun Y, Sun Z, Wang X, You J, Suo Y. Determination of triterpenic acids in fruits by a novel high performance liquid chromatography method with high sensitivity and specificity. Food Chem, 2014; 146:264269.

How to cite this article:

Wildner AC, Ferreira PL, Oliveira SS, Gnoatto SB, Bergold AM. Variation of Ursolic and Betulinic Acid in Five Malus $\times$ domestica clones from Southern Brazil. J App Pharm Sci, 2018; 8(09): 158-165. 


\section{Supplementary Material}

\section{Variation of Ursolic and Betulinic Acid in Five Malus $\times$ domestica clones from Southern Brazil}

Andreia C. Wildner ${ }^{1 *}$, Pâmela L. Ferreira ${ }^{1}$, Sendy S. Oliveira ${ }^{2}$, Simone B. Gnoatto ${ }^{1}$, Ana M. Bergold ${ }^{1}$

${ }^{1}$ Universidade Federal do Rio Grande do Sul/Programa de Pós-graduação em Ciências Farmacêuticas, Av. Ipiranga, 2752, Porto Alegre, RS 90610-000, Brazil.

${ }^{2}$ Universidade Federal do Rio Grande do Sul/Faculdade de Farmácia, Porto Alegre/RS, Brazil.
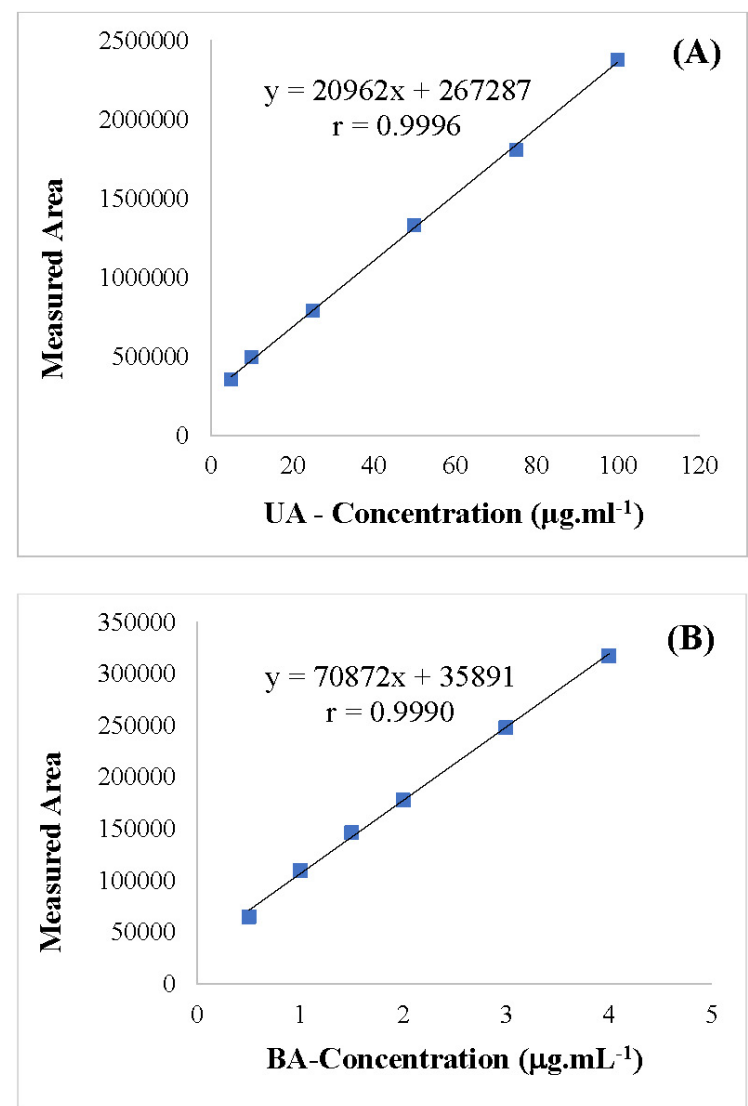

Fig. S1: Calibration curve of standards solution: a) UA concentrations from 5 to $100 \mu \mathrm{g} \cdot \mathrm{mL}^{-1}$; b) BA concentrations from 0.5 to $4 \mu \mathrm{g} \cdot \mathrm{mL}^{-1}$. 


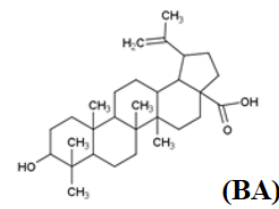

(BA)
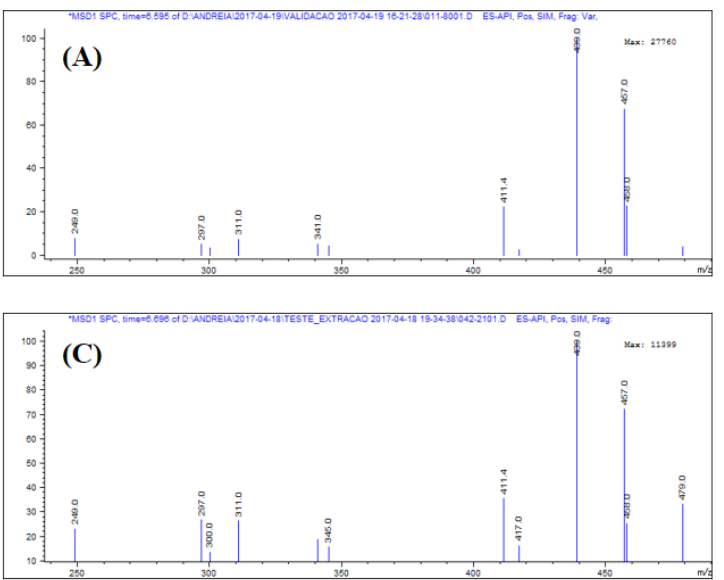
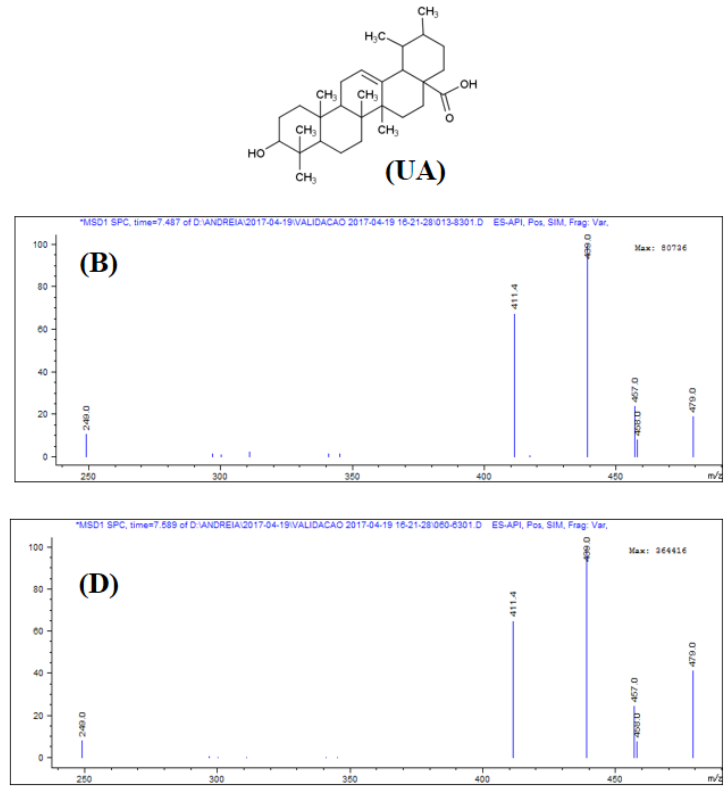

Fig. S2: MS spectra obtained by the analytical method developed, in SIM mode at $\mathrm{m} / z$ 457. (A) BA standard solution at $0.1 \mu \mathrm{g}$.mL $\mathrm{mL}^{-1}$, (B) UA standard solution at 0.1 $\mu \mathrm{g} \cdot \mathrm{mL}^{-1},(\mathrm{C})$ and (D) BA and UA in APE pool of Gala and Fuji cultivar, respectively.
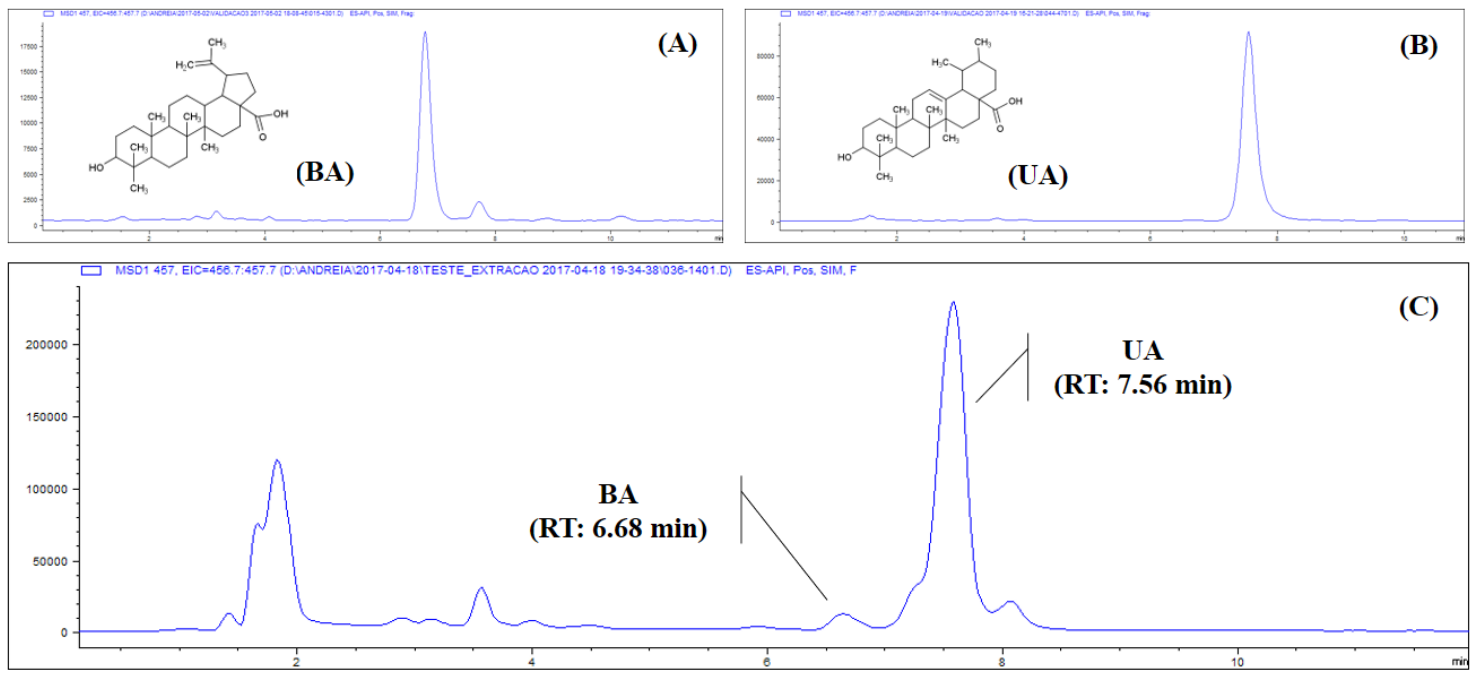

Fig. S3: LC-MS chromatograms obtained by the analytical method developed, in SIM mode at $m / z$ 457. (A) BA standard solution at $0.1 \mu$ g.mL ${ }^{-1}$, (B) UA standard solution at $0.1 \mu \mathrm{g} \cdot \mathrm{mL}^{-1},(\mathrm{C})$ and (D) BA and UA in APE pool of Gala and Fuji cultivar, respectively. 